\title{
A Dynamic Channel Allocation Mechanism for IEEE 802.11 Networks
}

\author{
Marcel William Rocha da Silva, José Ferreira de Rezende
}

\begin{abstract}
The great popularity of IEEE 802.11 along with channel scarcity may lead to a degraded overall performance if an intelligent channel allocation process is not used. A channel allocation unaware of other network's presence in the area may increase medium access contention and co-channel interference, harming networks' aggregate capacity. This work proposes a new automatic channel allocation mechanism for infra-structured IEEE 802.11 networks that works in an independent and distributed manner in access points and uses clients station's measurements exchanged with the new IEEE 802.11k standard.
\end{abstract}

\section{INTRODUCTION}

The growing number of networks based on wireless IEEE 802.11 standard has created some operational problems. The channel selection is one of the problems that appear in 802.11 networks operating in the infrastructure mode. At this mode, all packets are exchanged between stations and the Access Point (AP). The channel selection at the AP determines the center frequency utilized on these transmissions. This choice may adversely affect networks' performance by increasing the medium access contention and the co-channel interference.

The IEEE 802.11b [1] and 802.11g [2] standards operate in $2.4 \mathrm{GHz}$ unlicensed frequency band, known also as Industry, Scientific and Medical band (ISM band). IEEE 802.11 divides ISM band into 11 channels, but only 3 of them (channels 1 , 6 and 11) do not have spectrum overlap. IEEE 802.11a [3] operates in $5.4 \mathrm{GHz}$ unlicensed frequency band and offers 12 non-overlapping channels, 8 for indoor use and 4 for outdoor.

The 802.11 channel scarcity (a problem more evident in $802.11 \mathrm{~b}$ and $802.11 \mathrm{~g}$ standards) limits the number of networks that can coexist in the same area without interference. Spectrum sharing is the main source of interference, harming communication throughput and latency. However, other interference contributions, as adjacent channel interference, are also important. Hence, we can propose three basic objectives to decrease interference: spectral share minimization - avoiding medium access contention and maximizing frequency reuse; adjacent channel interference minimization - avoiding performance degradation generated by spectrum overlap; co-channel interference minimization - avoiding unacceptable signal-tonoise ratio (SNR) generated by remote stations transmissions.

In practice, the channel selection problem may appear in two different scenarios: with or without unique administration. In scenarios with unique administration, the central entity has

Marcel William Rocha da Silva and José Ferreira de Rezende are in the Grupo de Teleinformática e Automação, COPPE, Programa de Engenharia Elétrica, Universidade Federal do Rio de Janeiro, Rio de Janeiro-RJ, Brasil, 21.945-970, Phone: +55 212260 5010. Emails: \{marcel, rezende\}egta.ufrj.br. This work is supported by CAPES/CNPq/FINEP/FAPERJ. access to many important useful settings that make easier to find solutions and develop mechanisms to channel allocation problem. In scenarios of decentralized administration, different entities control the APs and there is very little knowledge about other APs in the area. These cases impose great challenges at the search of channel allocation solutions because of the information scarcity and the lack of coordination between the administrators controlling the APs.

This work proposes a new channel allocation mechanism for infrastructured 802.11 networks operating in decentralized scenarios. The proposed mechanism operates at the AP selecting the operating channel automatically and does not require communication between the APs. To achieve this goal, it uses some features proposed in the new IEEE 802.11k [4] draft standard that standardizes radio information measurements exchange amongst stations.

The next Section presents some channel selection related works and Section III explains some important 802.11 interference properties. Then, in Section IV, we present a brief overview of the new $802.11 \mathrm{k}$ draft standard. Sections V and VI present the proposed dynamic channel selection mechanism and its performance evaluation, respectively. Finally, Section VII draws the conclusions.

\section{RELATED WORKS}

Many other works have discussed the IEEE 802.11 channel allocation problem [5-9]. However, most of them analyze and propose solutions only to centralized scenarios. Solutions utilizing graph-coloring techniques are the most adopted [5, 6, $8,9]$. Usually, this approach considers each AP as a vertex of the network representing graph, and each potential interference region between the BSSs (Basic Service Sets) (Figure 1) as an edge.

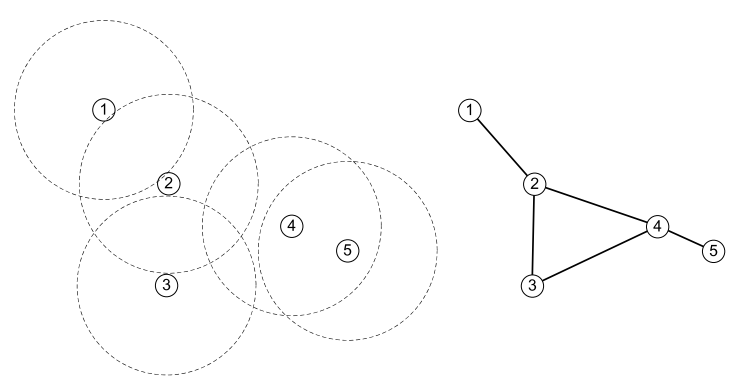

Fig. 1. Example of BSSs disposed in a region and its representing graph.

Finding graph coloring solutions to 802.11 channel allocation problem is, in general, a NP-hard problem $[6,8$, 9]. Therefore, algorithms to find optimal solutions present higher computational effort with larger networks. To face this 
limitation, the authors usually propose heuristics with low computational costs that find suboptimal solutions. The main difference between algorithms proposed for channel allocation is the objective function, which indicates what the coloring algorithms goal is.

The work [5] proposes a heuristic algorithm for graph coloring that takes into account the traffic demand of each BSS for the channel allocation. In [6], the authors proposed a greedy coloring algorithm to find a channel allocation that maximizes the number of neighbors with different channels. Work [7] claims that AP placement and channel selection are coupled problems and proposes a new technique to perform this task.

To the best of our knowledge, [8] is the only previous work to propose solution to channel allocation in decentralized scenarios. They developed a new approach to the coloring technique by setting weights to the edges of the interference graph indicating the relevance of utilizing different channels in the vertex connected by that edge. The edges weights are a metric of the number of stations placed in the conflict region that originated that edge. The BSSs' client stations are responsible for detecting the presence of other BSSs' client stations in interference region. They realize a passive scan of the operating channels to sense packets destined to other APs. The reason why client stations are responsible for this task, and not the AP, is that they have a better view of the interference scenario than the AP. Their proposed channel selection mechanism operates in centralized or distributed fashion.

The work in [8] was extended in [9], when the authors discuss that the channel selection task can be coupled with load balance. However, with this new increment the mechanism focus deviate from the decentralized scenario where the client stations cannot decide which AP it uses. Another channel selection mechanism, cited in [8] and [9], is the LCCS (Least Congested Channel Search). It is a mechanism implemented at some CISCO APs that searches for another channel (with less occupation) when others BSSs traffic exceeds a threshold.

The mechanism proposed in this paper resembles LCCS. The most important differences are that it measures others BSSs traffic at the client stations (which have a better picture of interference than the AP) and it searches better channels conditions proactively.

\section{INTERFERENCE IN IEEE 802.11}

Some points related to interference in 802.11 networks needs discussion for completely understanding of the proposed algorithm. Interference represents a problem in any wireless communication system. However, in 802.11 networks it appears in a slight different way, especially due to the medium access mechanism adopted by 802.11 .

With CSMA/CA, prior to transmitting a frame, a station senses the medium idle for a small amount of time. If it senses other stations' transmission, it waits the end of their transmission to start its own, avoiding a possible collision. This feature makes CSMA/CA very robust to interference in exchange to delay and throughput performance.
IEEE 802.11 transmissions generates three reception regions where receiving stations behave differently [10]. The reception zone, nearest to the transmitter, is where receiving stations can notice and decode packets. The carrier sense zone is the intermediate one, where receiving stations can only notice packets transmissions due to energy levels but they cannot be decoded. Moreover, at the interference zone, receiving stations cannot notice packet transmissions but their energy contribution is added to the noise floor as interference.

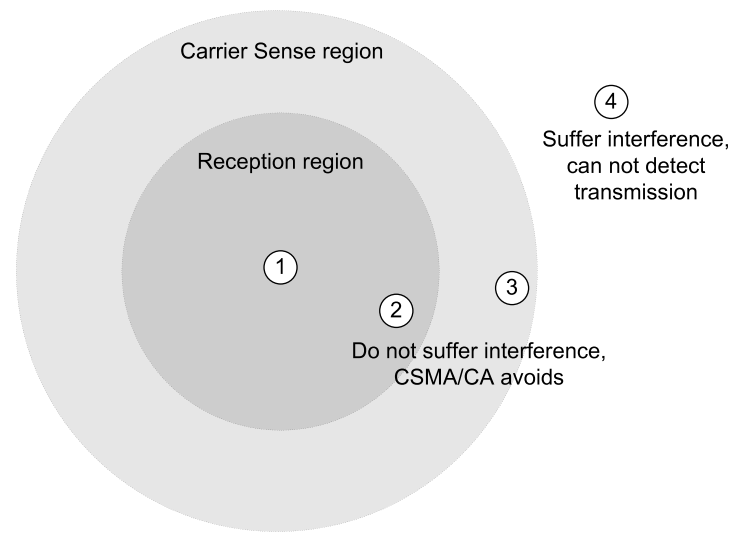

Fig. 2. CSMA/CA activity avoiding interference.

In Figure 2, when station (1) transmits, stations (2) and (3) must wait the end of this transmission before starting another one, sharing channel access with the station (1). Station (4), which is outside the reception and carrier sense zones, does not share medium access with station (1), but it suffers cochannel interference generated by its transmissions.

The above example shows that packet decoding (at reception zone) or carrier sense (at carrier sense zone) avoid concurrent transmissions of stations inside each other's reception or carrier sense zone. The packet decoding makes it obtain information about packet transmission duration (through a field of 802.11 MAC header). Therefore, stations set the NAV (Network Allocation Vector) that is an indicator counter of transmissions ending. The carrier sense itself detects that the medium is idle due to energy thresholds and avoids cochannel interference generation. This way, CSMA/CA avoids interference of near networks that are using the same channel. However, it increases medium access contention through medium access sharing.

As seen above, CSMA/CA particularities suggests that channel selection mechanism must differently account for near and far stations presence that increases medium access sharing or noise levels, respectively. In our proposal, we utilize channel load level and noise level to solve this problem.

\section{IEEE $802.11 \mathrm{~K}$ STANDARD}

The new $802.11 \mathrm{k}$ draft standard [4] specifies some types of radio information and the respective messages to request and report them. The main $802.11 \mathrm{k}$ objectives are to enable specific radio features measurements by network stations, standardize request/report messages to the exchange of these measurements and make the gathered information available to higher network layers. This standard development will offer 
useful management tools to network stations to provide useful information to new protocols and mechanisms.

\section{A. Radio Measurements}

So far, the $802.11 \mathrm{k}$ draft standard specifies eight types of radio measurements information. For the sake of brevity, we present only the radio measurements used in this proposal.

- Channel Load Information: gather channel load information in a specific number of channels in the measurement duration. The sensing station obtains it by dividing the amount of time the medium was idle by the measurement duration. The sensing station may use NAV or carrier sense to detect if the medium was idle.

- Noise Histogram: gives noise level histogram of noise values collected in a specific number of channels in the measurement duration. The sensing station makes noise measurements only when the medium is idle.

A station can conduct measurements at its operating channel or at non-serving channels ${ }^{1}$ and most of them may be performed passively only by packet exchange observation, without affecting network operation. However, non-serving channels measurement may lead to performance degradation since the sensing station needs to switch channels during measurement and stay incapable of exchanging packets during this period. The $802.11 \mathrm{k}$ standard recommends requesting only short and spaced non-serving channel measurements.

A request option that can reduce overhead generated by nonserving channels measuring is the concurrent measurement. The sensing node may conduct most information measurement concurrently, decreasing the information acquisition time and the performance harm it generates. Another feature that can reduce the impact of measuring non-serving channels is the packet buffering technique, which is already used in 802.11 power saving mechanism, where the AP stores packets destined to stations in power save mode. The stations could use this technique when measuring non-serving channels.

\section{B. Message Exchange}

IEEE $802.11 \mathrm{k}$ standard defines two basic message types: request and report messages. Stations may exchange these messages in station-station or station-AP fashion. Besides, they can send these messages in unicast, broadcast or multicast. The message exchange occurs either by one station requesting other station measurement information or by one station reporting by itself its measured information.

Each request/report message is a MAC management frame that has information about measurements settings, which advise the sensing station about how to execute measurements (channels, duration, start time, concurrency aspects, etc.). Each information measurement contains one element per information type that has the measurement information (in requests) or the measurement results (in reports).

${ }^{1}$ Channels different of the operating channel used by the network.

\section{Dynamic Channel SElection}

As discussed in Section III, a bad channel allocation may increase medium access contention and co-channel interference. Therefore, the channel selection algorithm proposed needs to consider this information at the selection process. To achieve this goal, we use the $802.11 \mathrm{k}$ standard to feed APs channel selection algorithm with channel load and noise levels. However, before presenting the algorithm, we present some concerns about how to obtain these inputs.

\section{A. Algorithm Inputs Acquisition}

With the proposed mechanism, client stations are responsible for conducting measurements. It is not desirable that the AP itself do measurements because client stations have a much better picture of potential interference problems than the AP $[8,9]$. Besides, making the AP switch channels periodically would turn down all BSS communications simultaneously.

The selection algorithm implemented in the AP uses request and report messages of the $802.11 \mathrm{k}$ standard in order to trigger and collect measurements. The AP periodically sends request messages to the client stations elected to perform the channels sensing task. These stations should perform measurements concurrently in all possible non-overlapping channels. Sensing nodes' election is an important step of the algorithm. We propose realizing measurements in all client stations since this generates a wide view of the interference problem.

The AP sends measurement requests to each station at a random time within the algorithm execution window. Hence, the algorithm will have reports from all client stations that it manages at its execution and will use the mean values of the reports received for load and noise levels.

One problem that should arise is the overhead generated by each client measuring all channels in each algorithm execution window. However, the overhead generated by this measurement only becomes significant if the measurements duration and periodicity are high. If these parameters are kept low, the overhead generated will be negligible.

Once it has measurement results from all channels, the channel selection algorithm starts its operation. The algorithm reads two arrays, one with channel load values and other with channel noise values. Each position carries the averages of channel load or noise level of the channel it represents. Obtaining each channel load value is a simple average calculation of the values returned by the client station reports. Obtaining noise average values, however, is not a simple average calculation since these values are returned in a histogram format (Figure 3). To obtain the average we used equation 1 .

\begin{tabular}{|l|l|l|l|l|}
\hline$a$ & $b$ & $c$ & $d$ & $e$ \\
\hline 1 & 2 & 3 & 4 & 5
\end{tabular}

Fig. 3. Histogram example: the letters represent the number of events at each value range, and the numbers represent the average value of each range.

$$
\text { Average }=\frac{1 \times a+2 \times b+3 \times c+4 \times d+5 \times e}{a+b+c+d+e}
$$


Note also that the channel load measurement should take into account only the traffic generated by others BSS stations. Otherwise, the AP can perform a channel switch when its own traffic raises channel occupation.

\section{B. Channel Selection Algorithm}

As the priority of the algorithm is to minimize channel load, it only considers noise levels of lightly loaded channels for selecting an operating channel. The algorithm has three steps:

1) if the load of the current channel does not exceed a certain threshold $\alpha$, the current operating channel is maintained and the algorithm is finished. Otherwise, proceeds to step 2 .

2) selects the $N$ channels with the lowest load and proceeds to step 3 .

3) chooses among the $N$ channels selected above the one that presents the lowest noise level.

The $\alpha$ threshold, cited in the first step, works like an aggressiveness adjust parameter, and indicates how much channel load is tolerated at the operating channel. Only if channel load exceeds the $\alpha$ parameter, the channel selection mechanism perform the search for a better condition executing steps two and three of the selection algorithm. This prevents many undesirable channel switches due to other BSS control traffic.

\section{Simulations}

For the evaluation of the automatic channel selection mechanism proposed, this work presents simulations with ns- $2^{2}$. The main objective of these simulations is to evaluate the algorithm parameters and the gains obtained with the channel selection mechanism.

The simulations with ns- 2 required the implementation of new features not present at its original code. Firstly, we implemented the channel occupation and noise measuring mechanisms as specified in the $802.11 \mathrm{k}$ standard. Besides, we also implemented all the co-channel noise calculation in ns-2. Other key feature implemented is the ability of switching channels during a simulation run. Moreover, in order to simulate infrastructured networks, we used the $\mathrm{NOAH}^{3}$ (No Ad-Hoc Routing Agent) module to avoid ad hoc routing traffic.

We fixed some parameters through the performed simulations. Each BSS comprehends one AP along with three client stations that generate one TCP flow each towards the AP (uplink traffic). The physical transmission rate is set to $11 \mathrm{Mbps}$ for data frames and $2 \mathrm{Mbps}$ for control frames.

\section{A. Channel Selection Interval}

An important parameter of the proposed mechanism is the channel selection interval. This parameter is a uniform random variable of a given mean value. For each simulation scenario, the mean value of the interval is varied from 20 seconds to 140 seconds, but keeping the same ratio between the mean

\footnotetext{
${ }^{2}$ The Network Simulator (ns-2) - http://www.isi.edu/nsnam/ns/

${ }^{3} \mathrm{NOAH}$ - http://icapeople.epfl.ch/widmer/uwb/ns-2/noah/
}

value, its upper and lower limits and the simulation duration. The uniform random variable limits are four times shorter than the mean, and the simulation duration is five times higher.

The simulations that evaluate the selection interval comprehend nine BSSs randomly disposed in a 2000-meter side square area, the $\alpha$ parameter is set to $10 \%$, the number of non-overlapping channels to three and the channel measuring time to $150 \mathrm{~ms}$. Fifty simulation runs for each selection interval were used. All results presented below are the averaged values of these runs with a $95 \%$ confidence interval.

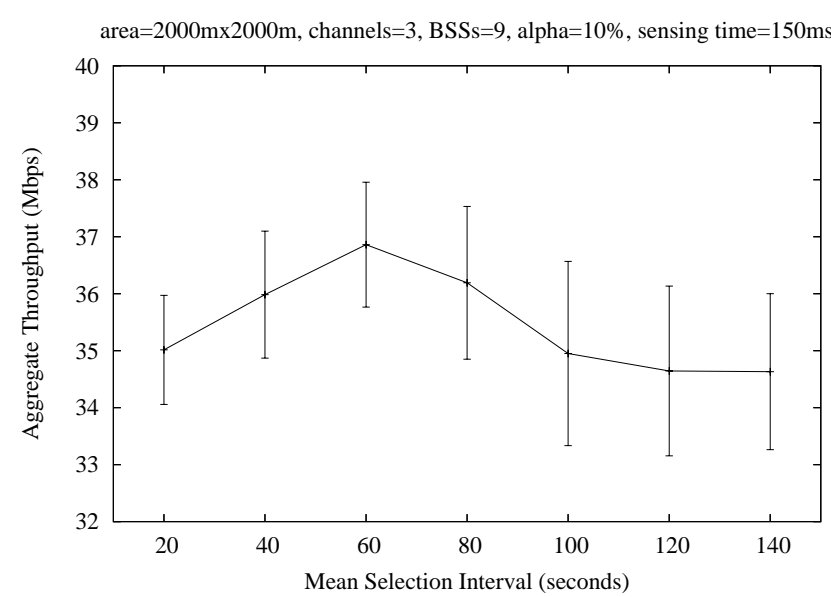

(a) Aggregate Throughput.

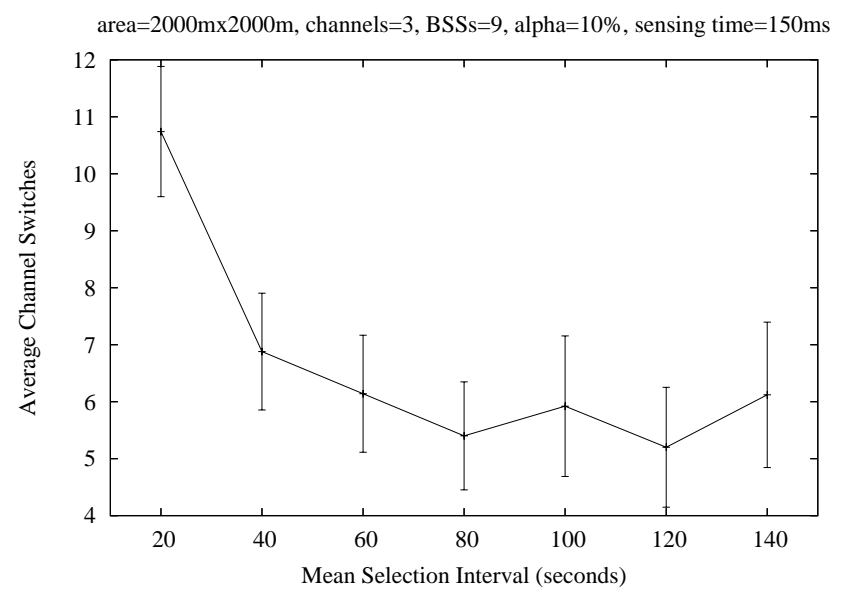

(b) Average Channel switches.

Fig. 4. Channel selection periodicity variation.

As it can be seen in Figure 4(a), the aggregate throughput presents some slight performance degradation for shorter and longer channel selection intervals. For shorter values, the harm generated by the communication pause time, which has a fixed value, increases. For longer channel selection intervals, the information measured by sensing nodes are outdated and do not reflect the real network interference picture, making the channel selection algorithm to perform wrong channel choices.

Figure 4(b) presents the number of channel switches during the simulation time. This value increases for short selection intervals. This happens because, as with the aggregate throughput, pauses in communication are more significant, affecting the sensing task, which may return wrong values. 
From the results, we conclude that for the channel selection interval a value about 60 seconds is the best choice for the scenarios analyzed. Therefore, we will use this value as a base to evaluate other algorithm parameters.

\section{B. Parameter $\alpha$}

For this evaluation, most simulation parameters are set as in the previous simulations, except the channel selection interval which is set to 60 seconds. Figures 5(a) and 5(b) present the aggregate throughput and the number of channel switches when the $\alpha$ parameter is varied from $0 \%$ to $100 \%$, respectively.

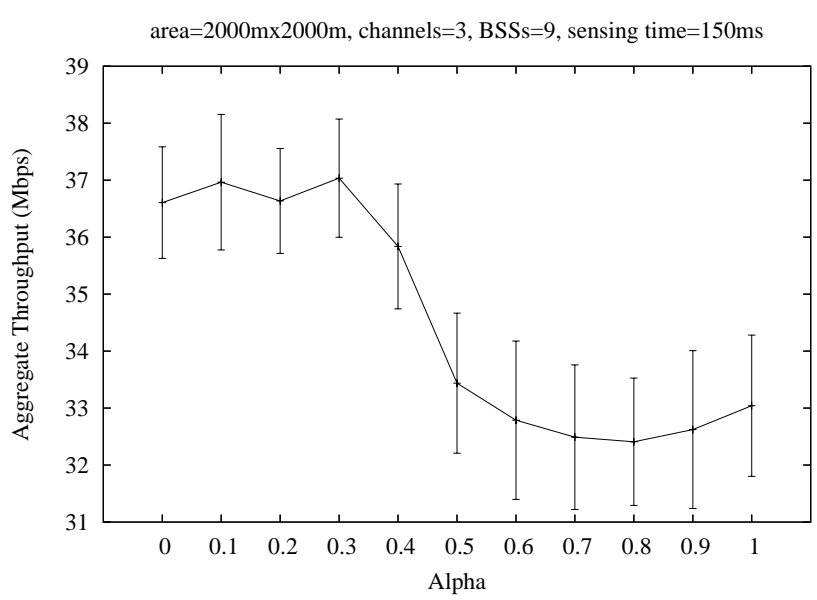

(a) Aggregate Throughput.

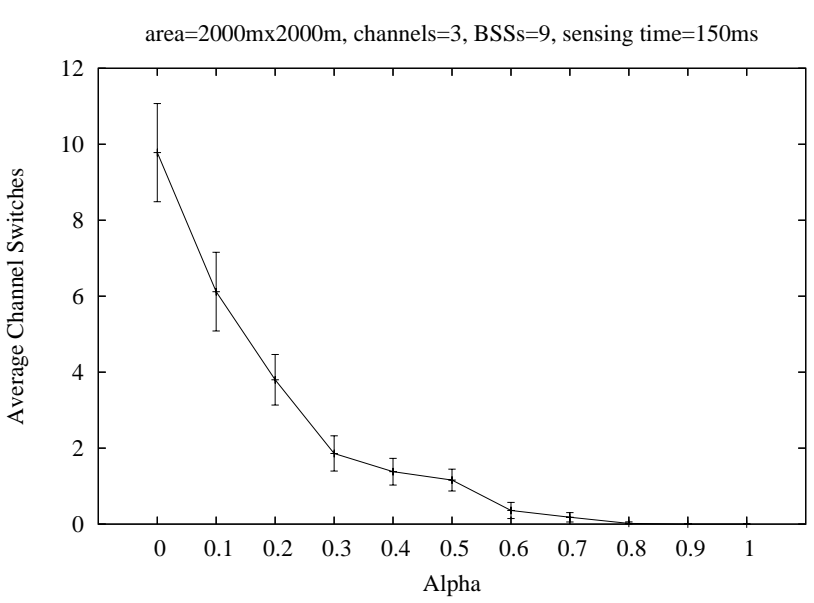

(b) Average channel switches.

Fig. 5. Variation of $\alpha$ parameter.

The results show that the aggregate throughput and the number of channel switches decay for higher $\alpha$ values. This happens because when $\alpha$ increases, it make the channel selection mechanism less aggressive, preventing it from performing channel switches. In other words, the parameter $\alpha$ accounts to how much interference the BSS may tolerate before performing a channel selection.

For the analyzed scenario, a good choice for this parameter would be something between $20 \%$ and $30 \%$, because, with this values, not much channel switches are needed by the selection mechanism to achieve a good network aggregate performance.

\section{Channel Sensing Time}

The channel measuring time may cause great influence over the performance of the selection algorithm since the amount of time spent sensing other channels also represents the amount of time that the client node is not allowed to transmit or receive its own packets. For the simulations, we disposed nine BSSs in the 2000-meter side square area and set the channel selection interval to 60 seconds and $\alpha$ to $10 \%$.

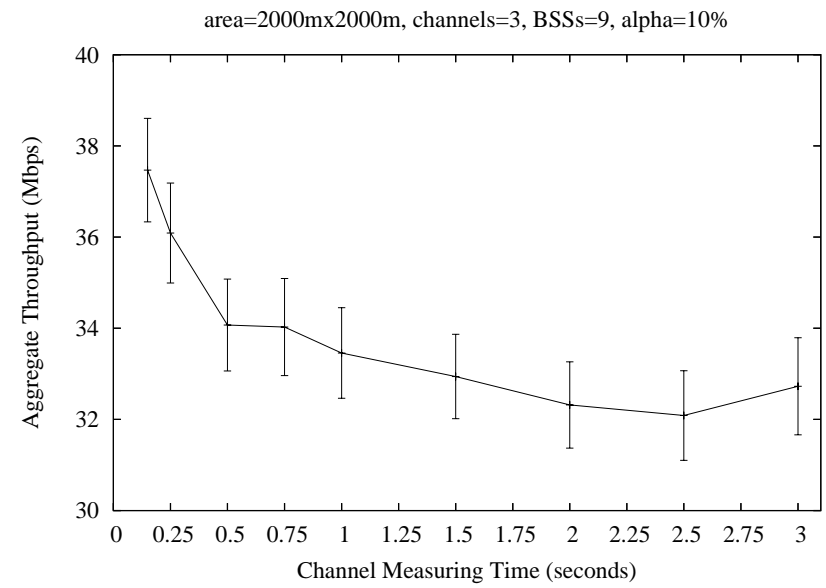

(a) Aggregate Throughput.

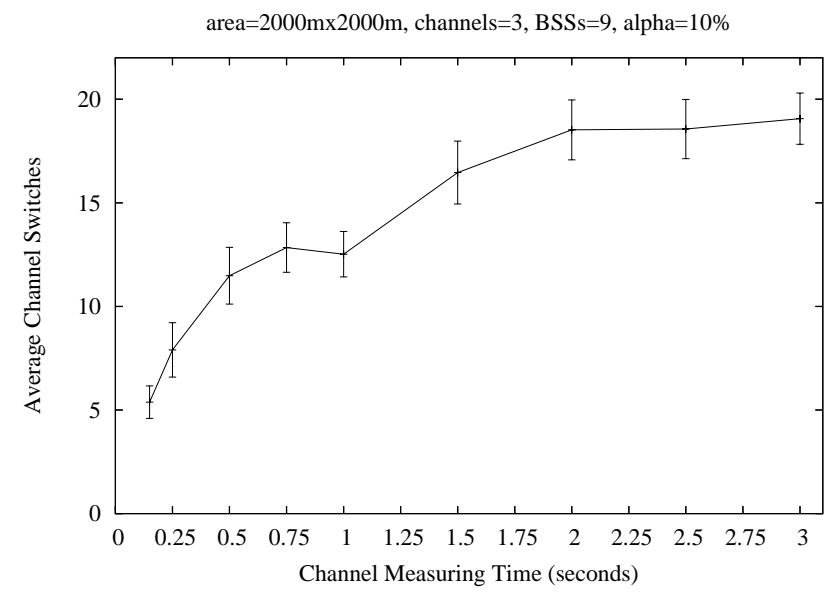

(b) Average Channel switches.

Fig. 6. Channel sensing time variation.

Figures 6(a) and 6(b) present the aggregate throughput and average number of channel switches when the channel measuring time is varied from $150 \mathrm{~ms}$ to 3 seconds, respectively. They show that higher measuring intervals decreases the aggregate throughput since the amount of time the client stays turned off increases. Moreover, it is important to remember that the measuring node needs to pause its communication twice the channel sensing interval when there are three non-overlapping channels. It also increases the number of channel switches because the increased sensing time started to affect the TCP traffic pattern harming the sensing task itself.

\section{Network Density}

In this scenario, we compare our algorithm to a random channel allocation algorithm when the density of BSSs dis- 
posed in a 2000-meter side square area and the number of non-overlapping channels are varied. The other parameters used were the suggested values obtained from the previous simulations. The channel selection interval is set to 60 seconds, the $\alpha$ is set to $30 \%$ and the channel sensing time is set to $150 \mathrm{~ms}$. Figures 7(a) and 7(b) present the results for aggregate throughput and average number of channel switches, respectively.

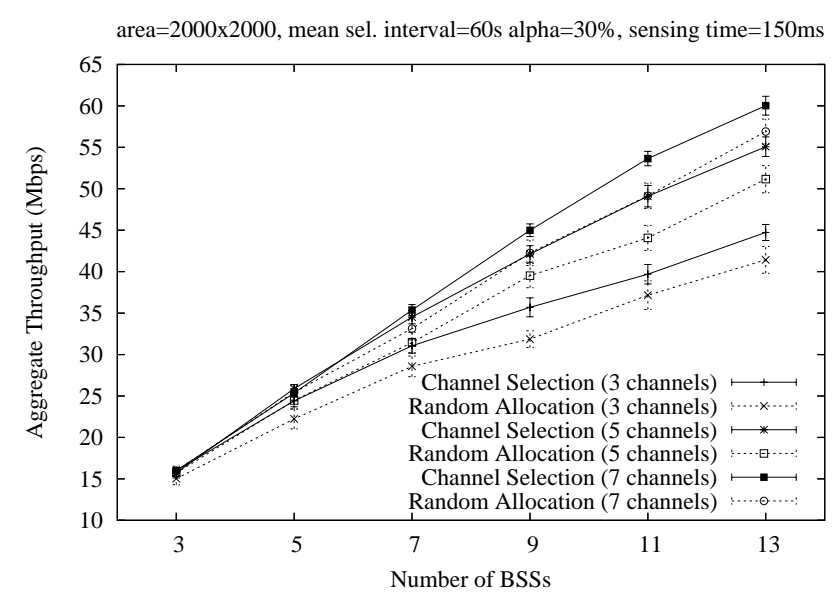

(a) Aggregate Throughput.

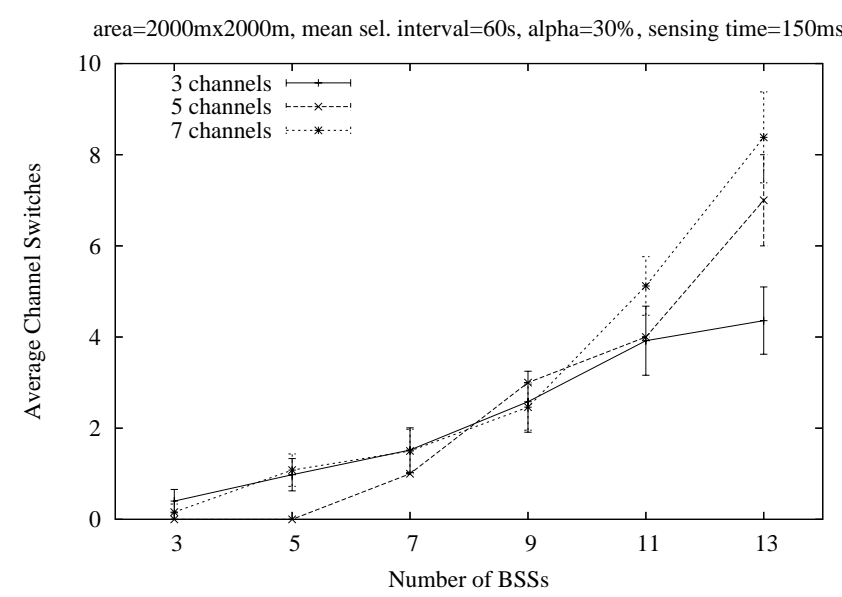

(b) Average Channel switches.

Fig. 7. Network density variation.

We may see from Figure 7(a) that the proposed mechanism performs better than the random channel allocation. With the channel selection mechanism turned on, the APs were able to search for better channel allocations, which mitigated interference and brought better throughputs. Furthermore, the gains of channel selection could be even better if we considered that in real scenarios without channel selection the allocation does not follow a uniform random distribution. Akella et al. [11] show that about $60 \%$ of the APs use channel 6 , which is the standard channel of some AP brands.

Figure 7(b) show that the average number of channel switches rises when more non-overlapping channels are available. However, it does not exceed one switch per BSS in average, showing that, in most simulations, a few channel switches were enough to achieve better channel allocations.

\section{CONCLUSIONS}

The non-centralized 802.11 scenarios along with channel scarcity pose challenges for the channel allocation problem. Without a central authority to decide or apply some channel allocation policy, the APs may experience great performance degradation. Therefore, the development of channel selection mechanisms, which are distributed and decentralized, is necessary to improve performance.

As expected, the channel selection mechanism proposed outperforms the random channel allocation. Moreover, it should present even higher gains if we compared our proposal with more realistic scenarios than the simple random allocation. Work [11], for instance, shows that in practice about $60 \%$ of the APs use channel 6. If we considered this distribution in our random allocation scenarios, they should perform even worse. The results for the average number of channel switches were also good. In most simulations, the selection algorithm needed an average of less than one switch per BSS to achieve better channel allocations.

\section{REFERENCES}

[1] 802.11b working group, "Wireless LAN medium access control (MAC) and physical layer (PHY) specifications: Higher-speed physical layer extension in the $2.4 \mathrm{ghz}$ band," 1999, IEEE Standard.

[2] 802.11g working group, "Wireless LAN medium access control (MAC) and physical layer (PHY) specifications: Further higher data rate extension in the $2.4 \mathrm{ghz}$ band," 2003, IEEE Standard.

[3] 802.11a working group, "Wireless LAN medium access control (MAC) and physical layer (PHY) specifications: High-speed physical layer in the 5 ghz band," 1999, IEEE Standard.

[4] 802.11k working group, "Wireless LAN medium access control (MAC) and physical layer (PHY) specifications: Radio resource measurement," 2005, IEEE Draft Standard.

[5] K. Leung and B.-J. Kim, "Frequency assignment for IEEE 802.11 wireless networks," in IEEE Vehicular Technology Conference, Oct. 2003.

[6] P. Mahonen, J. Riihijarvi, and M. Petrova, "Automatic channel allocation for small wireless local area networks using graph colouring algorithm approach," in IEEE International Symposium on Personal, Indoor and Mobile Radio Communications, Sept. 2004.

[7] X. Ling and K. L. Yeung, "Joint access point placement and channel assignment for 802.11 wireless lans," in IEEE Wireless Communications and Networking Conference (WCNC), Mar. 2005.

[8] A. Mishra, S. Banerjee, and W. Arbaugh, "Weighted coloring based channel assignment for WLANs," in Mobile Computing and Communications Review, July 2005.

[9] A. Mishra, V. Brik, S. Banerjee, A. Srinivasan, and W. Arbaugh, "A client-driven approach for channel management in wireless LANs," 2006, to appear in IEEE Conference on Computer Communications (INFOCOM).

[10] K. Xu, M. Gerla, and S. Bae, "How effective is the IEEE 802.11 RTS/CTS handshake in ad hoc networks?" in IEEE Global Telecommunications Conference, Nov. 2002.

[11] A. Akella, G. Judd, S. Seshan, and P. Steenkiste, "Self-management in chaotic wireless deployments," in International Conference on Mobile computing and networking (MOBICOM), Aug. 2005. 